\title{
Sur le principe du maximum et le balayage ${ }^{1)}$
}

\author{
Par Nobuyuki NinomiYa \\ (Reçu le 17 juillet, 1959)
}

\section{Introduction}

Dans le travail antérieur ([2]), on a étudié les problèmes de l'équilibre et du balayage pour les potentiels dont le noyau est une fonction $K(x, y)$ positive, continue et symétrique. On considère le potentiel d'une mesure positive $\mu$ pris par rapport au noyau $K$

$$
U^{\mu}(x)=\int K(x, y) d \mu(y)
$$

l'intégrale d'énergie d'une mesure positive $\mu$

$$
\|\mu\|^{2}=\int U^{\mu} d \mu
$$

et l'intégrale d'énergie mutuelle de deux mesures positives $\mu$ et $\nu$

$$
<\mu, \nu>=\int U^{\mu} d \nu=\int U^{\nu} d \mu .
$$

Considérons la quantité

$$
G(\mu, \nu)=\frac{\|\mu\|^{2} \times\|\nu\|^{2}}{\langle\mu, \nu\rangle^{2}}
$$

pour tout couple $(\mu, \nu)$ de deux mesures positives $(\neq 0) \mu$ et $\nu, \mu$ portée par $E_{1}, \nu$ portée par $E_{2}, E_{1}$ et $E_{2}$ étant deux ensembles boréliens et disjoints. $G(\mu, \nu)$ est bien déterminée seulement pour des couples $(\mu, \nu)$ tels que $\|\mu\|^{2},\|\nu\|^{2}$ et $\langle\mu, \nu\rangle^{2}$ tous les trois soient positifs et finis. En faisant des recherches des propriétés d'un couple $\left(\mu_{0}, \nu_{0}\right)$ qui rend minimum $G(\mu, \nu)$ parmi tous couples $(\mu, \nu)$, on a obtenu deux résultats suivants:

THÉoRÈme. Pour que le problème de l'équilibre soit résoluble pour un noyau $K$, il faut et il suffit que le noyau $K$ satisfasse au premier principe du maximum.

Theorème. Pour que le problème du balayage soit résoluble pour un noyau $K$, il faut et il suffit que le noyau $K$ satisfasse au second principe du maximum.

On a trouvé que tout noyau $K$ positif, continu et symétrique pour lequel le problème de l'équilibre ou du balayage est résoluble est toujours de type positif.

Dans le travail présent, on va étudier l'équilibre inverse, le balayage inverse et le balayage faible. Choquet-Deny ([1]) ont étudié dernièrement l'équilibre inverse et le balayage inverse et le balayage faible pour les potentiels dont le noyau est une

1) Ce travail a été rapporté à la séminaire de la théorie du potentiel à Paris organizée par MM. les Profs. Brelot, Choquet, et Deny au 29 avril, 1959. Son résumé sera publié dans le tirage de la séminaire de la théorie du potentiel à Paris pour l'année scolaire 1958-59. 
fonction positive définie dans l'espace de nombre fini des points. En approfondissant la quantité $G(\mu, \nu)$, ce travail établira dans l'espace plus général les résultats qu'ils ont obtenu dans l'espace de nombre fini des points, seulement lorsque le noyau est une fonction positive, continue et symétrique.

\section{L'équilibre inverse et le balayage inverse.}

On suppose que $K(x, y)$ est une fonction positive, bornée, continue et symétrique dans l'espace euclidien ou plus généralement dans l'espace topologique localement compact $\Omega$. Un noyau $K$ est dit satisfaire au principe du balayage inverse lorsque, pour tout compact $F$ et toute mesure positive $\mu$, il existe au moins une mesure positive $\mu^{\prime}$ portée par $F$ telle que

$$
\begin{array}{ll}
U^{\mu \prime}(x)=U^{\mu}(x) & \text { sur } F, \\
U^{\mu \prime}(x) \geqq U^{\mu}(x) & \text { dans tout l'espace. }
\end{array}
$$

Cette mesure $\mu^{\prime}$ est dite une mesure inversement balayée de $\mu$ sur $F$. Un noyau $K$ est dit satisfaire au second principe du minimum si, pour toute mesure positive $\mu$ à support compact $F$ et pour tout point $p$ n'appartenant pas à $F$, l'inégalité

$$
U^{\mu}(x) \geqq K(x, p)
$$

sur $F$ entraîne le même inégalité dans tout l'espace. Choquet-Deny ([1], p.97 et p.107) ont démontré qu'il y a équivalence entre le second principe du minimum et le principe du balayage inverse pour tout noyau qui est une fonction positive et finie dans l'espace de nombre fini des points. Plus généralement, on a

THÉORÈme 1. Pour qu'un noyau $K$ satisfasse au principe du balayage inverse, il faut et il suffit qu'il satisfasse au second principe du minimum. Les potentiels inversement balayés de toute mesure positive sur tout compact sont bien déterminés dans tout l'espace.

Pour démontrer le théorème, considérons

$$
G(\mu, \nu)=\frac{\|\mu\|^{2} \times\|\nu\|^{2}}{\langle\mu, \nu\rangle^{2}}
$$

pour tout couple $(\mu, \nu)$ de deux mesures positives $(\neq 0) \mu$ et $\nu$ portées par deux ensembles boréliens $E_{1}$ et $E_{2}$ respectivement. $K(x, y)$ étant borné et continu sur tout compact de $\Omega \times \Omega$, on peut considérer un couple qui rend maximum $G(\mu, \nu)$ parmi tous les couples $(\mu, \nu)$. Alors,

LEMME 1. S'il existe un couple maximal $\left(\mu_{0}, \nu_{0}\right)$, on a

$$
\begin{array}{ll}
g_{1}(x) \leqq 0 & \text { sur } E_{1}, \\
g_{1}(x) \geqq 0 & \text { sur le support de } \mu_{0},
\end{array}
$$

où

$$
\begin{aligned}
& a=\left\|\mu_{0}\right\|^{2}, \quad b=\left\|\nu_{0}\right\|^{2}, \quad c=<\mu_{0}, \nu_{0}>, \\
& g_{1}(x)=c U^{u_{0}}(x)-a U^{\nu_{0}}(x) \quad \text { et } \quad g_{2}(x)=c U^{\nu_{0}}(x)-b U^{u_{0}}(x) .
\end{aligned}
$$

Résultats analogues pour $g_{2}(x)$.

En effet, on a la relation

$$
G\left(\mu_{0}+\sigma, \nu_{0}\right) \leqq G\left(\mu_{0}, \nu_{0}\right)
$$


pour toute mesure $\sigma$ de signe quelconque portée par $E_{1}$ telle que $\mu_{0}+\sigma$ soit positive. Elle entraîne l'inégalité

$$
0 \geqq 2 c \int g_{1} d \sigma+\left[c^{2}\|\sigma\|^{2}-a<\nu_{0}, \sigma>^{2}\right]
$$

On peut obtenir facilement les propriétés (1) et (2) en prenant une mesure convenable pour $\sigma$ comme on l'a déjà montré ([2], p.151).

Remarque. Si $E_{1}$ et $E_{2}$ tous les deux sont des compacts, il existe toujours un couple maximal $\left(\mu_{0}, \nu_{0}\right)$ de $G(\mu, \nu)$.

En effet, si on pose

$$
G^{*}=\sup G(\mu, \nu),
$$

il existe deux suites de mesures $\left\{\mu_{n}\right\}$ portées par $E_{1}$ et de mesures $\left\{\nu_{n}\right\}$ portées par $E_{2}$ telles que

$$
\lim _{n \rightarrow \infty} G\left(\mu_{n}, \nu_{n}\right)=G^{*} .
$$

Comme on l'a déjà dit ([2], p.152), on peut supposer que toute $\mu_{n}$ et toute $\nu_{n}$ sont de masse totale un, et donc que $\left\{\mu_{n}\right\}$ et $\left\{\nu_{n}\right\}$ convergent vers une mesure positive $\mu_{0}$ portée par $E_{1}$ et vers une mesure positive $\nu_{0}$ portée par $E_{2}$ respectivement, en prenant leurs suites partielles convergeants à l'avance. Le noyau $K$ étant borné et continu, on a

$$
\lim _{n \rightarrow \infty}\left\|\mu_{n}\right\|^{2}=\left\|\mu_{0}\right\|^{2}, \quad \lim _{n \rightarrow \infty}\left\|\nu_{n}\right\|^{2}=\left\|\nu_{0}\right\|^{2}
$$

et

$$
\lim _{n \rightarrow \infty}\left\langle\mu_{n}, \nu_{n}\right\rangle=\left\langle\mu_{0}, \nu_{0}\right\rangle .
$$

Ainsi, $\left(\mu_{0}, \nu_{0}\right)$ est un couple maximal.

En fixant une mesure $\nu$ portée par $E_{2}$ dans $G(\mu, \nu)$, considérons

$$
G(\mu)=\frac{\|\mu\|^{2}}{\langle\mu, \nu\rangle^{2}}
$$

pour toute mesure positive $(\neq 0) \mu$ portée par un compact $E_{1}$. Alors, on a de la même manière que le lemme 1

Corollaire. Une mesure $\mu_{0}$ qui rend maximum $G(\mu)$ parmi toutes les mesures positives $(\equiv 0) \mu$ portées par un compact $E_{1}$ jouit des propriétés suivantes:

$$
\begin{array}{ll}
g(x) \leqq 0 & \text { sur } E_{1}, \\
g(x) \geqq 0 & \text { sur le support de } \mu_{0}
\end{array}
$$

où $a=\left\|\mu_{0}\right\|^{2}, c=\left\langle\mu_{0}, \nu_{0}\right\rangle$ et $\dot{g}(x)=c U^{\mu_{0}}(x)-a U^{\nu}(x)$.

Démonstration du théorème 1 . Supposons qu'un noyau $K$ satisfait au principe $\mathrm{du}$ balayage inverse. Soient $p$ un point quelconque et $\mu$ une mesure positive dont le support $F$ est un compact ne contenant pas $p$, telle que

$$
U^{\mu}(x) \geqq K(x, p)
$$

sur $F$. Si on désigne par $\varepsilon_{x}^{\prime}$ une mesure inversement balayée de la mesure ponctuelle $\varepsilon_{x}$ placée en un point $x$ sur $F$, on a

$$
\begin{aligned}
U^{\mu}(x) & =\left\langle\varepsilon_{x}, \mu>=\left\langle\varepsilon_{x^{\prime}}, \mu>=\left\langle\mu, \varepsilon_{x}^{\prime}\right\rangle\right.\right. \\
& \left.\geqq<\varepsilon_{p}, \varepsilon_{x}^{\prime}\right\rangle=\left\langle\varepsilon_{x^{\prime}}, \varepsilon_{p}>\geqq<\varepsilon_{x}, \varepsilon_{p}>=K(x, p) .\right.
\end{aligned}
$$


Donc, le noyau $K$ satisfait au second principe du minimum. Inversement, supposons qu'un noyau $K$ satisfait au second principe du minimum. Soient $F$ un compact et $p$ un point n'appartenant pas à $F$. Pour construire une mesure inversement balayée de $\varepsilon_{p}$ sur $F$, prenons une mesure $\mu_{0}$ qui rend maximum

$$
G(\mu)=\frac{\| \mu_{\|}^{2}}{<\mu, \varepsilon_{p}>^{2}}
$$

parmi toutes les mesures positives $(\neq 0) \mu$ portées par $F$. En posant

$$
a=\left\|\mu_{0}\right\|^{2}, \quad c=<\mu_{0}, \varepsilon_{p}>\text { et } \varepsilon_{p}^{\prime}=\frac{c}{a} \mu_{0},
$$

on a

$$
\begin{aligned}
& U^{\varepsilon} p^{\prime}(x) \leqq K(x, p) \quad \text { sur } F, \\
& U^{\varepsilon_{p}}(x) \geqq K(x, p) \quad \text { sur le support de } \varepsilon_{p^{\prime}} .
\end{aligned}
$$

Le second principe du minimum entraine que $\varepsilon_{p}^{\prime}$ est une mesure inversement balayée de $\varepsilon_{p}$ sur $F$. Ensuite, soit $p$ un point de $F$. Pour construire une mesure inversement balayée de $\varepsilon_{p}$ sur $F$, prenons une suite de voisinages $\left\{G_{n}\right\}$ de $p$ tels que $G_{n} \downarrow p$. Alors, la limite $\varepsilon_{p}^{\prime}$ de la suite des mesure inversement balayées $\varepsilon_{p_{n}}{ }^{\prime}$ de $\varepsilon_{p}$ sur $F_{n}=F-G_{n}$ est une mesure inversement balayée de $\varepsilon_{p}$ sur $F$. Pour construire une mesure inversement balayée d'une mesure positive quelconque $\mu$ sur $F$, prenons une suite des mesures ponctuelles $\mu_{n}$ convergeant vers $\mu$. Alors, la limite $\mu^{\prime}$ de la suite des mesures inversement balayées $\mu_{n}^{\prime}$ de $\mu_{n}$ sur $F$ est une mesure inversement balayée de $\mu \operatorname{sur} F$. Enfin, soient $\mu^{\prime}$ et $\mu^{\prime \prime}$ tous les deux des mesures inversement balayées d'une mesure positive $\mu$ sur un compact $F$. Pour toute mesure positive $\nu$ et pour une mesure inversement balayée $\nu^{\prime}$ de $\nu$ sur $F$, on a

$$
\left\langle\mu^{\prime}, \nu\right\rangle=\left\langle\mu^{\prime}, \nu^{\prime}\right\rangle=\left\langle\mu^{\prime \prime}, \nu^{\prime}\right\rangle=\left\langle\mu^{\prime \prime}, \nu\right\rangle \text {. }
$$

Donc, on a dans tout l'espace

$$
U^{\mu \prime}(x)=U^{\mu \prime \prime}(x) .
$$

Ainsi, les potentiels inversement balayés de $\mu$ sur $F$ sont bien déterminés, bien que des mesures inversement balayées de $\mu$ sur $F$ ne soient pas uniques.

Un noyau $K$ est dit satisfaire au principe de l'équilibre inverse lorsque, pour tout compact $F$, il existe au moins une mesure positive $\lambda$ portée par $F$ telle que

$$
\begin{array}{ll}
U^{x}(x)=1 & \text { sur } F, \\
U^{\lambda}(x) \geqq 1 & \text { dans tout l'espace. }
\end{array}
$$

Cette mesure $\lambda$ est dite une mesure inversement équilibrée sur $F$ et le potentiel $U^{\lambda}$ est dit un potential inversement équilibré sur $F$. Un noyau $K$ est dit satisfaire au premier principe du minimum si, pour toute mesure positive $\lambda$ à support compact $F$, l'inégalité

$$
U^{2}(x) \geqq 1
$$

sur $F$ entraîne la même inégalité dans tout l'espace. Alors, on a

Theorème 2. Pour qu'un noyau $K$ satisfaise au principe de l'équilibre inverse, il faut et il suffit que le noyau $K$ satisfasse au premier principe du minimum.

Démonstration. Supposons qu'un noyau $K$ satisfait au premier principe du minimum. Etant donné un compact $F$, il existe une mesure $\mu_{0}$ qui rend maximum l'intégrale d'énergie 


$$
I=\|\mu\|^{2}
$$

parmi toutes les mesures positives $\mu$ portées par $F$ de masse totale un, car $K(x, y)$ est borné et continu sur tout compact de $\Omega \times \Omega$. On a l'inégalité

$$
\left\|\mu_{0}+\sigma\right\|^{2} \leqq\left\|\mu_{0}\right\|^{2}
$$

pour toute mesure $\sigma$ de masse totale nulle telle que $\mu_{0}+\sigma$ soit une mesure positive portée par $F$, ce qui entraîne facilement, en posant

$$
\begin{array}{cl}
\lambda=\frac{\mu_{0}}{\left\|\mu_{0}\right\|^{2}}, \\
U^{\lambda}(x) \leqq 1 & \text { sur } F, \\
U^{\lambda}(x) \geqq 1 & \text { sur le support de } \lambda .
\end{array}
$$

Cette mesure $\lambda$ est une mesure inversement équilibrée sur $F$ en vertu du premier principe du minimum. Ensuite, supposons qu'un noyau $K$ satisfait au principe de l'équilibre inverse. Soit $\lambda$ une mesure positive à support compact $F$ telle que

$$
U^{2}(x) \geqq 1
$$

sur $F$. S'il y avait un point $p$ tel que $U^{2}(p)<1$, prenons une mesure $\mu_{0}$ qui rend maximum

$$
G(\mu)=\frac{\|\mu\|^{2}}{\left\langle\mu, \varepsilon_{p}\right\rangle^{2}}
$$

parmi toutes les mesures positives $(\neq 0) \mu$ portées par $F$. On peut supposer que la masse totale de $\mu_{0}$ est un. Alors, en posant

$$
a=\left\|\mu_{0}\right\|^{2}, \quad c=<\mu_{0}, \varepsilon_{p}>\text { et } g(x)=c U^{\mu_{0}}(x)-a K(x, p)
$$

on a en vertu du corollaire du lemme 1

$$
0 \geqq \int g(x) d \lambda(x)=c<\mu_{0}, \lambda>-a<\varepsilon_{p}, \lambda>>c-a .
$$

D'autre part, en désignant par $\lambda_{0}$ une mesure inversement équilibrée sur le support $F_{0}$ de $\mu_{0}$, on a

$$
0 \leqq \int g(x) d \lambda_{0}(x)=c<\mu_{0}, \lambda>-a<\varepsilon_{p}, \lambda_{0}>\leqq c-a,
$$

ce qui est contradictoire. Par suite, on a

$$
U^{2}(x) \geqq 1
$$

dans tout l'espace.

Un noyau $K$ positif, borné, continu et symétrique est dit de type négatif lorsqu'on a l'inégalité

$$
\|\mu\|^{2} \times\|\nu\|^{2} \leqq<\mu, \nu>^{2}
$$

pour tout couple $(\mu, \nu)$ de deux mesures positives $\mu$ et $\nu$. Alors, on a

THÉORÈme 3. Tout noyau satisfaisant au principe de l'équilibre inverse ou $d u$ balayage inverse (c'est-a-dire, au premier ou second principe du minimum) est toujours de type négatif.

Démonstration. Soient $E_{1}$ et $E_{2}$ des compacts quelconques et $\left(\mu_{0}, \nu_{0}\right)$ un couple qui rend maximum

$$
G(\mu, \nu)=\frac{\|\mu\|^{2} \times\|\nu\|^{2}}{\langle\mu, \nu\rangle^{2}}
$$


parmi tous les couples $(\mu, \nu), \mu$ portées par $E_{1}$ et $\nu$ portées par $E_{2}$. On a en vertu du lemme 1

$$
\begin{array}{ll}
c U^{\mu_{0}}(x) \geqq a U^{\nu_{0}}(x) & \text { sur le support de } \mu_{0}, \\
c U^{\nu_{0}}(x) \geqq b U^{\mu_{0}}(x) & \text { sur le support de } \nu_{0} .
\end{array}
$$

Si le noyau $K$ satisfait au premier principe du minimum, on a

$$
\begin{aligned}
\frac{c}{a} \inf _{x \in S \mu_{0}} U^{\mu_{0}}(x) & \geqq \inf _{x \in S \mu_{0}} U^{\nu_{0}}(x) \geqq \inf _{x \in S \nu_{0}} U^{\nu_{0}}(x) \\
& \geqq \frac{b}{c} \inf _{x \in S \nu_{0}} U^{\mu_{0}}(x) \geqq \frac{b}{c} \inf _{x \in S \mu_{0}} U^{\mu_{0}}(x) .
\end{aligned}
$$

Par suite, on a

$$
G\left(\mu_{0}, \nu_{0}\right)=\frac{a b}{c^{2}} \leqq 1
$$

Si le noyau $K$ satisfait au second principe du minimum, on a deux inégalités (1) et (2) dans tout l'espace. Par suite, on a

$$
G\left(\mu_{0}, \nu_{0}\right)=\frac{a b}{c^{2}} \leqq 1
$$

Donc, on a toujours

$$
G(\mu, \nu) \leqq 1
$$

pour tout couple $(\mu, \nu)$.

Lemme 2. Soit $K$ un noyau de type négatif. Si on a

$$
G\left(\mu_{0}, \nu_{0}\right)=1
$$

pour un couple $\left(\mu_{0}, \nu_{0}\right)$ de deux mesures positives $\mu_{0}$ et $\nu_{0}$, on a

$$
g_{1}(x)=g_{2}(x)=0
$$

dans tout l'espace, où

$$
\begin{aligned}
a & =\left\|\mu_{0}\right\|^{2}, \quad b=\left\|\nu_{0}\right\|^{2}, \quad c=<\mu_{0}, \nu_{0}>, \\
g_{1}(x) & =c U^{\mu_{0}}(x)-a U^{\nu_{0}}(x) \quad \text { et } \quad g_{2}(x)=c U^{\nu_{0}}(x)-b U^{\mu_{0}}(x) .
\end{aligned}
$$

En effet, les inégalités

$$
G\left(\mu_{0}+\sigma, \nu_{0}\right) \leqq G\left(\mu_{0}, \nu_{0}\right)
$$

et

$$
G\left(\mu_{0}, \nu_{0}+\tau\right) \leqq G\left(\mu_{0}, \nu_{0}\right)
$$

ayant lieu pour toutes mesures positives $\sigma$ et $\tau$ à support compact entraînent

$$
g_{1}(x) \leqq 0 \text {. et } g_{2}(x) \leqq 0
$$

dans tout l'espace. D'autre part, on a l'égalité

$$
c g_{1}(x)+a g_{2}(x)=0
$$

dans tout l'espace, d'où le resultat.

THÉoRème 2 (bis). Soit $K$ un noyau satisfaisant au principe de l'équilibre inverse. Les potentiels inversement équilibrés sur tout compact sont bien déterminés dans tout l'espace.

En effet, soient $\lambda_{1}$ et $\lambda_{2}$ des mesures inversement équilibrées sur un compact $F$. Comme on a 


$$
G\left(\lambda_{1}, \lambda_{2}\right)=1
$$

on a en vertu du lemme 2

$$
<\lambda_{1}, \lambda_{2}>U^{\lambda_{1}}(x)-\left\|\lambda_{1}\right\|^{2} U^{\lambda_{2}}(x)=0
$$

dans tout l'espace. Comme on a

on a d'ailleurs

$$
<\lambda_{1}, \lambda_{2}>=\left\|\lambda_{1}\right\|^{2}
$$

dans tout l'espace.

$$
U^{\lambda_{1}}(x)=U^{\lambda_{2}}(x)
$$

\section{Le balayage faible.}

On considère dans ce paragraphe un noyau $K(x, y)$ qui est une fonction positive, continue et symétrique dans l'espace topologique localement compact $\Omega$ et qui pourra être $+\infty$ en $x=y$. On doit supposer que tout ensemble ouvert de $\Omega$ n'est pas de $K$-capacité nulle. ${ }^{2)}$ Un noyau $K$ est dit satisfaire au principe du balayage faible lorsque, pour toute mesure positive $\mu$ et pour tout compact $F$, il existe au moins une mesure positive $\mu^{\prime}$ portée par $F$ telle que

$$
U^{\mu \prime}(x)=U^{\mu}(x)
$$

sur $F$ hors d'un ensemble de $K$-diamètre transfini nul. ${ }^{2)}$ Cette mesure $\mu^{\prime}$ est dite une mesure faiblement balayée de $\mu$ sur $F$ et le potentiel $U^{\mu \prime}$ est dit un potentiel faiblement balayé de $\mu$ sur $F$. Et un noyau $K$ est dit satisfaire au principe de l'équilibre faible lorsque, pour tout compact $F$, il existe au moins une rnesure positive $\lambda$ portée par $F$ telle que

$$
U^{2}(x)=1
$$

sur $F$ hors d'un ensemble de $K$-diamètre transfini nul. On va travailler les relations entre le balayage (ou l'équilibre) faible et le balayage (ou l'équilibre) ordinaire ou inverse. Evidemment, tout noyau qui satisfait au principe du balayage (ou l'équilibre) ordinaire ou inverse satisfait au principe du balayage (ou l'équilibre) faible. D'autre part, on a

THÉORÈme 4. Si un noyau positif et symétrique $K$ est de type positif, le principe $d u$ balayage faible entraine le principe $d u$ balayage ordinaire et le principe de l'équilibre faible entraîne le principe de l'équilibre ordinaire. Si un noyau positif et symétrique $K$ est de type négatif, le principe du balayage faible entraîne le principe du balayage inverse et le principe de l'équilibre faible entraîne le principe de l'équilibre inverse.

Lemme 3. Si un noyau $K$ satisfait au principe du balayage faible, il satisfait au second principe du maximum faible; pour toute mesure positive $\mu$ a support compact $F$ et pour tout point $p$ n'appartenant pas a $F$, si on a

$$
U^{\mu}(x) \leqq K(x, p)
$$

sur $F \cup\{p\}$, on a la même inégalité dans tout l'espace.

En effet, si $K(p, p)<+\infty$, l'ensemble $\{p\}$ est de $K$-diamètre transfini positif.

2) Un ensemble borélien est dit de $K$-capacité nulle s'il ne porte aucune mesure positive dont le potentiel est borné dans tout compact, et est dit de $K$-diamètre transfini nul s'il ne porte aucune mesure positive dont l'intégrale d'énergie est finie. 
Soit $\varepsilon_{x}^{\prime}$ une mesure faiblement balayée de la mesure ponctuelle $\varepsilon_{x}$ placée en un point $x$ sur $F \cup\{p\}$. Les mesures $\mu$ et $\varepsilon_{p}$ étant tous les deux d'énergie finie, on a

$$
U^{\mu}(x)=\left\langle\varepsilon_{x}, \mu\right\rangle=\left\langle\mu, \varepsilon_{x}^{\prime}\right\rangle \leqq\left\langle\varepsilon_{p}, \varepsilon_{x}^{\prime}\right\rangle=\left\langle\varepsilon_{x^{\prime}}{ }^{\prime}, \varepsilon_{p}\right\rangle=K(x, p) .
$$

Si $K(p, p)=+\infty$, soit $\nu$ une mesures positive d'énergie finie, de masse totale un et portée par un voisinage $G$ de $p$ telle qu'on aie

$$
U^{\mu}(x) \leqq U^{\nu}(x)+\eta
$$

sur $F \cup G$, $\eta$ étant un assez petit nombre positif donné. Soit $\varepsilon_{x}^{\prime}$ une mesure faiblement balayée de $\varepsilon_{x}$ sur $F \cup G$. Les mesures $\mu$ et $\nu$ étant tous les deux d'énergie finie, on a

$$
U^{\mu}(x)=\left\langle\mu, \varepsilon_{x}^{\prime}\right\rangle \leqq<\nu, \varepsilon_{x}^{\prime}>+\eta \cdot \int d \varepsilon_{x^{\prime}}=U^{\nu}(x)+\eta \cdot \int d \varepsilon_{x^{\prime}} .
$$

Comme la mesure $\nu$ est arbitrairement rapprochée à $\varepsilon_{p}, \eta$ est arbitrairement petit et la masse totale de $\varepsilon_{x}^{\prime}$ est évidemment bornée, on a

$$
U^{\mu}(x) \leqq K(x, p)
$$

dans tout l'espace.

Lemme 4. Si un noyau $K$ satisfait au principe de l'équilibre faible, il satisfait au principe de masse totale faible; pour toute mesure positive $\mu$ à support compact $F$ et pour toute point p n'appartenant pas à $F$, si on a

$$
U^{\mu}(x) \leqq K(x, p)
$$

sur $F \cup\{p\}$, on $a$

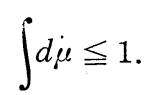

En effet, si $K(p, p)<+\infty$, soit $\lambda$ une mesure faiblement équilibrée sur $F \cup\{p\}$. Les mesures $\mu$ et $\varepsilon_{p}$ étant tous deux d'énergie finie, on a

$$
\int d \mu=\left\langle\lambda, \mu>=\langle\mu, \lambda\rangle \leqq\left\langle\varepsilon_{p}, \lambda\right\rangle=\left\langle\lambda, \varepsilon_{p}\right\rangle=1 .\right.
$$

Si $K(p, p)=+\infty$, soit $\nu$ une mesure positive d'énergie finie, de masse totale un et portée par un voisinage $G$ de $p$ telle qu'on aie

$$
U^{\mu}(x) \leqq U^{\nu}(x)+\eta
$$

sur $F \cup G, \eta$ étant un assez petit nombre positif donné. Soit $\lambda$ une mesure faiblement équilibrée sur $F \cup G$. Alors, on a

$$
\int d \mu=\left\langle\lambda, \mu>\leqq<\lambda, \nu>+\eta \cdot \int d \lambda=\int d \nu+\eta \cdot \int d \lambda\right.
$$

Comme la mesure $\nu$ est arbitrairement rapprochée à $\varepsilon_{p}, \eta$ est arbitrairement petit et la masse totale dè $\lambda$ est évidemment bornée, on a

$$
\int d \mu \leqq 1
$$

Démonstration du théorème 4. Supposons qu'un noyau $K$ soit de type positif et satisfasse au principe du balayage faible. Soient $F$ un compact de $K$-diamètre transfini positif et $p$ un point n'appartenant pas à $F$. Considérons

$$
G(\mu)=\frac{\|\mu\|^{2}}{<\mu, \varepsilon_{p}>^{2}}
$$

pour toute mesure positive $(\not \equiv 0) \mu$ portée par $F$. Soit $\mu_{0}$ une mesure qui rend mini- 
mum $G(\mu)$ parmi toutes les mesures positives portées par $F$. Si on pose

$$
a=\left\|\mu_{0}\right\|^{2} \quad \text { et } \quad c=\left\langle\mu_{0}, \varepsilon_{p}\right\rangle
$$

on a transfini nul,

$$
c U^{\mu_{0}}(x) \geqq a K(x, p) \quad \text { sur } F \text { hors d'un ensemble de } K \text {-diamètre }
$$

$$
c U^{\mu_{0}}(x) \leqq a K(x, p) \quad \text { sur le support de } \mu_{0} .
$$

La dernière relation (2) a lieu aussi en le point $x=p$, car le noyau $K$ est de type positif et on a

$$
G\left(\mu_{0}\right) \cdot K(p, p) \geqq 1
$$

D'après le second principe du maximum faible, on a en posant $\varepsilon_{p}{ }^{\prime}=(c / a) \mu_{0}$ transfini nul,

$$
U^{\varepsilon} p^{\prime}(x) \leqq K(x, p) \quad \text { dans tout l'espace. }
$$

Ce montre que cette $\varepsilon_{p}{ }^{\prime}$ est une mesure balayée de $\varepsilon_{p}$ sur $F$. Pour démontrer que le noyau $K$ satisfait au principe du balayage ordinaire, il suffit de démontrer qu'il satisfait au second principe du maximum. Soient $\mu$ une mesure positive d'énergie finie à support compact $F$ et $\nu$ une mesure positive telle que

$$
U^{\mu}(x) \leqq U^{\nu}(x)
$$

$\operatorname{sur} F$. Pour tout point $p$,en utilisant la mesure $\varepsilon_{p}{ }^{\prime}$ ci-dessus, on a

$$
U^{\mu}(p)=\left\langle\varepsilon_{p}{ }^{\prime}, \mu\right\rangle=\left\langle\mu, \varepsilon_{p}{ }^{\prime}\right\rangle \leqq<\nu, \varepsilon_{p}{ }^{\prime},>=\left\langle\varepsilon_{p}{ }^{\prime}, \nu>\leqq U^{\nu}(p) .\right.
$$

Ainsi, on sait que le principe du balayage faible entraîne le principe du balayage ordinaire, pourvu que le noyau $K$ soit de type positif. Ensuite, supposons qu'un noyau $K$ soit de type positif et satisfasse au principe de l'équilibre faible. Soient $F$ un compact de $K$-diamètre transfini positif $p$ et un point n'appartenant pas à $F$. Considérons

$$
G(\mu)=\frac{\|\mu\|^{2}}{<\mu, \varepsilon_{p}>^{2}}
$$

pour toute mesure positive $(\neq 0) \mu$ portée par $F$. Soit $\mu_{0}$ une mesure qui rend minimum $G(\mu)$ parmi toutes les mesures positives portées par $F$. Si on pose

$$
a=\left\|\mu_{0}\right\|^{2}, \quad c=<\mu_{0}, \varepsilon_{p}>\quad \text { et } \quad \varepsilon_{p^{\prime}}^{\prime}=\frac{c}{a} \mu_{0}
$$

on a les relations mentionées ci-dessus

(1) $\quad U^{\varepsilon_{p^{\prime}}}(x) \geqq K(x, p) \quad$ sur $F$ hors d'un ensemble de $K$-diamètre transfini nul,

$$
U^{\varepsilon} p^{\prime}(x) \leqq K(x, p) \quad \text { sur le support de } \varepsilon_{p}{ }^{\prime} \text { et en } x=p .
$$

D'après le principe de masse totale, la dernière relation (2) entraîne

$$
\int d \varepsilon_{p}^{\prime} \leqq 1 \text {. }
$$

Pour démontrer que le noyau $K$ satisfait au principe de l'équilibre, il suffit de démontrer qu'il satisfait au premier principe du maximum. Soit $\lambda$ une mesure positive à support compact $F$ telle que

$$
U^{\dot{\lambda}}(x) \leqq 1
$$


sur $F$. S'il y avait un point $p$ où $U^{\lambda}(p)>1$, on a en prenant la mesure $\varepsilon_{p}^{\prime}$ mentionée ci-dessus

$$
1<U^{\lambda}(p)=\left\langle\lambda, \varepsilon_{p}>=\left\langle\varepsilon_{p}, \lambda>\leqq<\varepsilon_{p}{ }^{\prime}, \lambda>=<\lambda, \varepsilon_{p}{ }^{\prime}\right\rangle \leqq \int d \varepsilon_{p}{ }^{\prime} \leqq 1,\right.
$$

ce qui est contradictoire. Donc, on a

$$
U^{\lambda}(x) \leqq 1
$$

dans tout l'espace. Ainsi on sait que le principe de l'équilibre faible entraîne le principe de l'équilibre ordinaire pourvu que le noyau $K$ soit de type positif. Au cas où un noyau $K$ est de type négatif, on peut démontrer de façon analogue que le principe du balayage faible (ou l'équilibre faible) entraîne le principe du balayage (ou l'équilibre) ordinaire.

Dans leur étude des modèles finis ([2]), Choquet et Deny ont montré que, si un noyau positif $K$ satisfait au principe du balayage faible, il satisfait, soit au principe du balayage ordinaire, soit au principe du balayage inverse. La question se pose de la validité de ce résultat dans un espace localement compact quelconque.

Question: Y-a-t'il un noyau positif et symétrịque qui satisfait au principe du balayage faible ou au principe de l'équilibre faible, mais qui n'est ni de type postif, ni de type négatif?

\section{Université Municipale d'Osaka.}

\section{Bibliographie}

[1] Choquet, G., et J. Deny, Modèles finis en théorie du potentiel. J. Analyse Math. Jerusalem 5 (1956-57), 77-135.

[2] Ninomiya, N., Etude sur la théorie du potentiel pris par rapport au noyau symétrique. J. Inst. Polytech. Osaka City Univ., Series A 8 (1957), 147-179. 